\title{
Transplacental sildenafil rescues lung abnormalities in the rabbit model of diaphragmatic hernia
}

\author{
Francesca M Russo, ${ }^{1,2}$ Jaan Toelen, ${ }^{1,3}$ M Patrice Eastwood, ${ }^{1}$ Julio Jimenez, ${ }^{1,4}$ \\ Andre Hadyme Miyague, ${ }^{1}$ Greetje Vande Velde, ${ }^{5}$ Philip DeKoninck, ${ }^{1}$ \\ Uwe Himmelreich, ${ }^{3}$ Patrizia Vergani, ${ }^{6}$ Karel Allegaert, ${ }^{1,7}$ Jan Deprest ${ }^{1,2,8}$
}

\begin{abstract}
- Additional material is published online only. To view please visit the journal online (http://dx.doi.org/10.1136/ thoraxjn-2015-207949)

${ }^{1}$ Cluster Organ Systems, Department of Development and Regeneration, Biomedical Sciences, KU Leuven, Leuven, Belgium

${ }^{2}$ Department of Obstetrics and Gynecology, University Hospitals Leuven, Leuven, Belgium

${ }^{3}$ Department of Pediatrics, University Hospitals Leuven, Leuven, Belgium

${ }^{4}$ Department of Obstetrics and Gynecology, Clinica Alemana, Santiago, Chile

${ }^{5}$ Department of Imaging and Pathology, Biomedical MRI/MoSAIC, KU Leuven, Leuven, Belgium

${ }^{6}$ Department of Obstetrics and Gynecology, University of Milano-Bicocca, Monza, Italy ${ }^{7}$ Department of Neonatalogy, University Hospitals Leuven, Leuven, Belgium

${ }^{8}$ Department of Obstetrics and Gynecology, Institute of Women's Health, University College London, London, UK
\end{abstract}

\section{Correspondence to}

Professor Jan Deprest, Fetal Medicine Unit, Division of Woman and Child, Department of Obstetrics and Gynaecology, University Hospitals Leuven, Herestraat 49, Leuven 3000, Belgium;

jan.deprest@uzleuven.be

Received 20 October 2015 Revised 17 February 2016 Accepted 20 February 2016 Published Online First

17 March 2016

\section{CrossMark}

\footnotetext{
To cite: Russo FM,

Toelen J, Eastwood MP,

et al. Thorax 2016;71:

517-525.
}

\section{ABSTRACT}

Introduction The management of congenital diaphragmatic hernia (DH) would benefit from an antenatal medical therapy, which addresses both lung hypoplasia and persistent pulmonary hypertension. We aimed at evaluating the pulmonary effects of sildenafil in the fetal rabbit model for $\mathrm{DH}$.

Methods We performed a dose-finding study to achieve therapeutic fetal plasmatic concentrations without toxicity following maternal sildenafil administration. Subsequently, DH fetuses were randomly exposed to transplacental placebo or sildenafil $10 \mathrm{mg} / \mathrm{kg} /$ day from gestational day 24 until examination at term (day 30). Efficacy measures were ipsilateral pulmonary vascular and airway morphometry, micro-CT-based branching analysis, Doppler flow in the main pulmonary artery and postnatal lung mechanics.

Results Fetal sildenafil plasmatic concentration was above the minimal therapeutic level for at least $22 \mathrm{~h} /$ day without maternal and fetal side effects. The placeboexposed DH fetuses had increased wall thickness in peripheral pulmonary vessels and significantly less fifth-order vessels compared with controls (CTR). Sildenafil-exposed DH fetuses, instead, had a medial and adventitial thickness in peripheral pulmonary vessels in the normal range and normal vascular branching. Fetal pulmonary artery Doppler showed a reduction of pulmonary vascular resistances both in DH and in CTR fetuses treated by sildenafil compared with the placebotreated ones. Sildenafil also reversed the mean terminal bronchiolar density to normal and improved lung mechanics, yet without measurable impact on lung-to-bodyweight ratio.

Conclusions In the rabbit model for $\mathrm{DH}$, antenatal sildenafil rescues vascular branching and architecture, reduces pulmonary vascular resistances and also improves airway morphometry and respiratory mechanics.

\section{INTRODUCTION}

The prevalence of congenital diaphragmatic hernia (DH) ranges between 1 and 4/10 000 births. This means that in Europe around 2000 children are born with this condition every year (2008). ${ }^{1}$ Lung development is first hit already in the embryonic period, but is further disturbed by lack of space when viscera herniate through the defect into the chest. As a consequence, DH lungs have fewer and less mature airway branches, a smaller crosssectional area of pulmonary vessels with structural remodelling and altered vasoreactivity. ${ }^{2}$ At birth,

\section{Key messages}

What is the key question?

- Data from rodent studies suggest that antenatal sildenafil could attenuate the lung vascular changes, which lead to pulmonary hypertension in infants affected by congenital diaphragmatic hernia, but the safety and efficacy of the drug still need to be validated in a higher animal model.

\section{What is the bottom line?}

- In the rabbit model for diaphragmatic hernia, maternally administered sildenafil reverses all the pathological changes in lung peripheral vessels and also results in a morphological and functional improvement in lung parenchyma without obvious fetal and maternal toxicity, except for fetuses with normally developed lungs in whom it seems to decrease vascular branching.

\section{Why read on?}

- This study provides proof of the efficacy and safety of antenatal sildenafil in rabbit fetuses with experimental diaphragmatic hernia, and paves the way for translation towards clinical evaluation, which eventually may reduce the high mortality and morbidity related to this condition.

these changes lead to respiratory insufficiency and persistent pulmonary hypertension (PPHT). ${ }^{3}$ The latter is lethal in up to $30 \%$, despite prenatal referral to high volume centres that offer standardised neonatal care. ${ }^{4} 5$ Survivors may suffer from additional morbidities such as chronic PPHT in up to $50 \%$ of cases, chronic lung disease, gastrooesophageal reflux and, later in life, thoracic deformations. ${ }^{6}$

Prenatal medical imaging methods can be used to identify future non-survivors ${ }^{7}$ and therefore offer the potential for prenatal interventions that could avoid that outcome. Fetoscopic endoluminal tracheal occlusion (FETO) is an investigational minimally invasive procedure that prevents egress of lung liquid, causing increased pulmonary stretch leading to accelerated lung growth. ${ }^{8}$ Observational studies have shown an apparent increase in survival and 
reduced early neonatal respiratory morbidity compared with historical controls (CTR). ${ }^{9}$ Still, the procedure leads to preterm delivery in $25 \%$ of cases and the maximum post-FETO survival is $50 \%-60 \%$. The remainder mortality is caused by insufficient airway growth and/or limited vascular development. Therefore, complementary or preferentially alternative non-invasive therapeutic treatments would be welcomed. These should ideally address both the vascular and airway problems in the disease.

The phosphodiesterase type 5 (PDE5) inhibitor sildenafil induces pulmonary vascular dilatation and pulmonary angiogenesis, and it inhibits pulmonary artery remodelling and perivascular smooth muscle proliferation. ${ }^{10}$ It has been approved by the Food and Drug Administration (FDA) for use in pulmonary hypertension in adults ${ }^{11}$ and often used as second-line therapy in PPHT in the newborn. ${ }^{12}$ Furthermore, clinical trials have been recently approved to evaluate its effect in pregnancies with severe intrauterine growth restriction. ${ }^{13}$ The drug has also already been considered for prenatal treatment of DH. In the rat model, maternally administered sildenafil improved all pathological features which are the basis for PPHT, without measurable side effects in other PDE5-expressing organs. ${ }^{14}$ However, before these findings can be translated into the clinical setting, a study in a larger animal model with a lung development more similar to that of man is needed. In this study, we assessed the safety and transplacental passage of sildenafil and evaluated its efficacy in the rabbit DH model.

\section{METHODS}

This study was approved by the Ethics Committee on Animal Experimentation of the Faculty of Medicine, KU Leuven, Leuven, Belgium (Project number: P014/2013) and follows the Animal Research: Reporting of In Vivo Experiments (ARRIVE) guidelines for reporting animal research.

Below is a summary of the Methods; additional details can be found in the online supplementary material.

\section{Determination of the therapeutic interval}

Total target sildenafil concentration (calculated as sildenafil concentration $+50 \%$ of its active $\mathrm{N}$-desmethyl metabolite DM-sildenafil concentration ${ }^{15}$ ) was between 47 and $500 \mathrm{ng} /$ $\mathrm{mL}$, according to previously published literature. Peak concentrations in excess of $500 \mathrm{ng} / \mathrm{mL}$ in humans have been previously shown to cause a $40 \%$ incidence of visual disturbances and $25 \%$ of vascular events. ${ }^{16}$ A target of $47 \mathrm{ng} / \mathrm{mL}$ was selected so that the concentration of unbound sildenafil would be expected to produce a $53 \%$ inhibition of PDE5 activity. ${ }^{17}$ This was also the target concentration used in the trial for FDA approval of treatment of pulmonary arterial hypertension in children. ${ }^{18}$

\section{Dose-finding and tolerance study}

The optimal sildenafil dose defined by a pilot study (described in details in the online supplementary material) was tested in a larger sample size to study the kinetics and assess the safety of the drug. Does underwent a sham laparotomy on gestational day (GD)23 (term $=31$ days) to take into account the effects of surgery on tolerance indices. After the operation, does were randomly assigned to two groups, receiving either placebo or sildenafil $10 \mathrm{mg} / \mathrm{kg} /$ day for a maximum of 7 days (from GD24 to GD30) (figure 1A). In the sildenafil group, does were terminally anaesthetised at 30 and $60 \mathrm{~min}, 6,12$ and $22 \mathrm{~h}$ after the first injection and an additional time point $22 \mathrm{~h}$ after the third injection, and maternal blood samples were collected by central ear artery puncture. Immediately after maternal euthanasia, three randomly chosen fetuses from each doe were delivered via caesarean section and anaesthetised with $200 \mu \mathrm{L}$ ketamine given intraperitoneally. Fetal blood samples were obtained by cardiac puncture. Blood samples were centrifuged at $3000 \mathrm{rpm}$ for $15 \mathrm{~min}$, frozen and analysed for sildenafil and DM-sildenafil by high-performance liquid chromatography with mass spectrometric detection (VU Medisch Centrum Laboratory for Clinical Pharmacology and Pharmacy, Amsterdam, The Netherlands). ${ }^{19}$

Remaining does were monitored daily for tolerance indices until harvest at GD31. These were maternal weight change percentage $(\mathrm{WCP}=$ (body weight before therapy-body weight at end of therapy)/body weight at end of therapy $\times 100$ ); changes from baseline in behaviour, assessed by a combination of validated behavioural scales ${ }^{20} 21$ (see online supplementary table S1); heart rate change percentage $(\mathrm{HRCP}=(\mathrm{HR}$ before the sham laparotomy-HR before the harvest)/HR before the harvest $\times 100$ ) monitored by a pulse oximeter. Fetal tolerance indices were fetal body weight (FBW) and fetal loss rate.

\section{Efficacy study}

Time-mated does underwent feto-maternal surgery on GD23 for induction of left-sided $\mathrm{DH}$ as previously described. ${ }^{22}$ In each doe, a maximum of three fetuses were operated, that is, on the ovarian ends and one additional fetus at least one gestational sac away from the operated fetuses. After surgery, does were randomly assigned to receive a daily injection of either placebo or the selected dose of sildenafil from GD24 until GD30 by using randomisation software (GraphPad Software). Tolerance indices were recorded till GD31, when does were euthanised to harvest the fetuses. Non-operated littermates of comparable size to the operated ones served as CTR.

\section{Gross anatomy}

Gross anatomy was evaluated in fetuses further used for lung morphometry analysis. Fetuses were delivered $20 \mathrm{~min}$ after maternal sacrifice to ensure fetal death and prevent fetal breathing. FBW and total fetal lung weight were measured, allowing calculation of the lung-to-body weight ratio (LBWR).

\section{Airway and vascular morphometry}

Fetal lungs and the trachea were removed en bloc and processed as described in the online supplementary material ${ }^{23}$ for histological and immunohistochemical analysis.

\section{Vascular architecture assessed by micro-CT scans}

Details on the method for in vivo barium perfusion and CT scan acquisition are provided in the online supplementary material. The vessel volume/total volume ratio was calculated based on full stack histograms of a manually delimited volume of interest. ${ }^{24}$ Finally, manual counting of vessel branching and order on reconstructed three-dimensional (3D) images was performed. ${ }^{25}$

\section{Fetal ECG}

Fetal ECG was performed on GD30.5, $3 \mathrm{~h}$ after the last dose of sildenafil. The VisualSonics VEVO 2100 (Toronto, Canada) high-resolution microultrasound platform and a VisualSonics MS-250 transducer (13-24 MHz, VisualSonics) were used for data acquisition, which was done in accordance with the American Society of Echocardiography guidelines and standards for performance of the fetal ECG. ${ }^{26}{ }^{27} \mathrm{M}$-mode ECG indices and Doppler measurements are described in the online supplementary material. 
Figure 1 Kinetics and tolerance of sildenafil $10 \mathrm{mg} / \mathrm{kg} / \mathrm{day}$. (A) Timeline of the dose-finding and tolerance study. (B) Maternal and fetal plasma total sildenafil levels at different time points. Error bars indicate SDs. The dotted lines depict the therapeutic range. (C) Mean course of behavioural score by gestational age. There were significant differences to baseline at gestational day (GD)24-26, but both groups were equally affected. Squares indicate mean behavioural scores; error bars indicate SD. Letters indicate post hoc corrected $p$ values of comparisons to baseline values; sildenafil:

$a: p=0.035, b: p=0.019, c: p=0.008$ $d: p=0.019$, e: $p=0.003$.
A

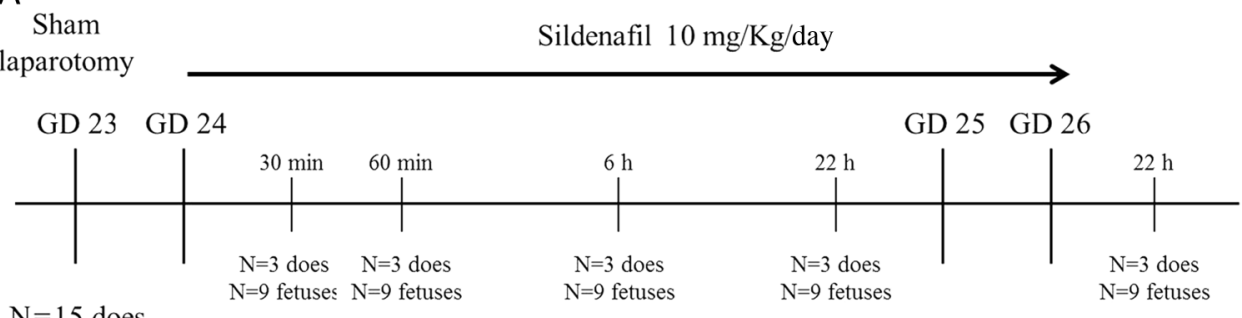

$\mathrm{N}=15$ does

B

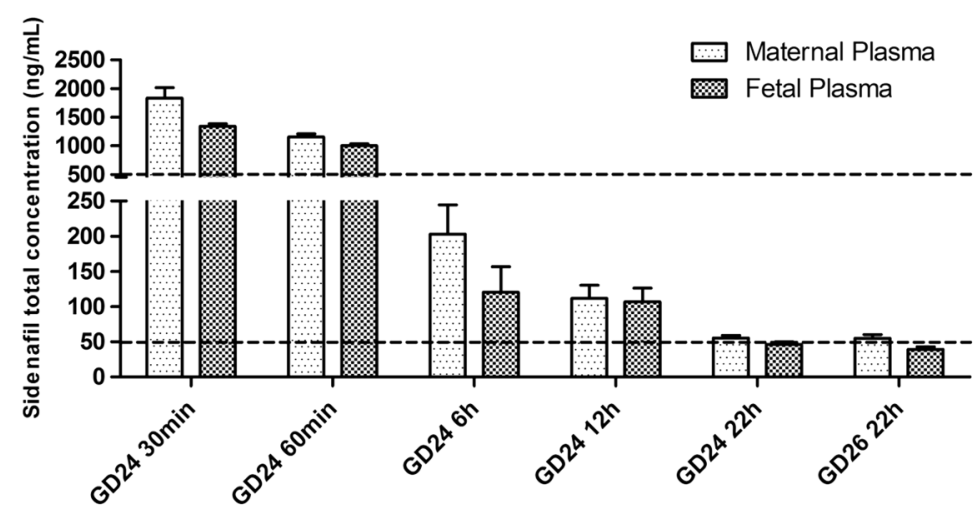

C

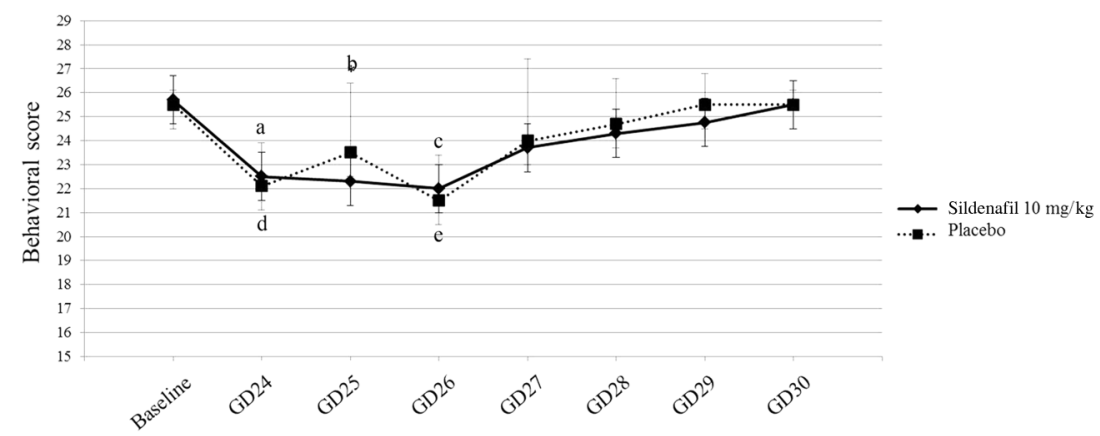

\section{Measurements of ventilatory mechanics}

Lung function was assessed in fetuses used previously for ECG measurements. After ultrasound examination of selected fetuses, involved fetuses were delivered via caesarean section for lung function testing as earlier described. ${ }^{28}$ The hysterotomy was sutured to prevent excessive bleeding and the does were maintained under general anaesthesia until the extraction of the last selected fetus, after which they were euthanised.

Invasive lung function testing was performed after 3-5 min of spontaneous breathing using a forced oscillation technique with the FlexiVent system (FlexiVent; SCIREQ, Montreal, Canada).

\section{Statistics}

According to the previously published literature, ${ }^{29}$ we determined that a sample size of five and six fetuses/group would provide a power of $\geq 90 \%$ with a two-sided type I error of $5 \%$ to detect a $10 \%$ reduction in proportionate medial thickness (\%MT) and a 20\% reduction in mean terminal bronchiolar density (MTBD) respectively.

The Kolmogorov-Smirnov test was used to assess the distribution of variables. Those with a normal distribution are presented as mean $\pm S D$ and $t$ test or analysis of variance combined with Tukey's multiple comparisons test were used to evaluate differences between treatment groups. Variables not normally distributed were expressed as median and IQR and were analysed using the Wilcoxon rank-sum test. A detailed overview of individual outcome measures and statistical tests is provided in the online supplementary table S2. A 2-tailed $\mathrm{p}<0.05$ was considered significant. All comparisons were performed using Prism for Windows V.5.0 (GraphPad software, San Diego, California).

\section{RESULTS}

Sildenafil crosses the placenta and reaches stable fetal levels without inducing fetal toxicity

Figure $1 \mathrm{~B}$ shows maternal and fetal total plasma concentration profiles after subcutaneous administration of $10 \mathrm{mg} / \mathrm{kg}$ sildenafil starting on GD24. This dose led to therapeutic fetal plasmatic concentrations for at least $22 \mathrm{~h} /$ day. Sildenafil kinetics was

Table 1 Tolerance parameters in placebo-treated and sildenafil-treated animals

\begin{tabular}{lccl}
\hline & Placebo & Sildenafil $10 \mathrm{mg} / \mathrm{kg} /$ day & $\mathrm{p}$ Value \\
\hline WCP & $3.8 \pm 1.9$ & $0.4 \pm 6.9$ & 0.3 \\
HRCP & $-6.8 \pm 18.25$ & $-6.0 \pm 21.2$ & 0.9 \\
Mean fetal loss rate (\%) & $9.1 \pm 5.1$ & $8.2 \pm 12.7$ & 0.7 \\
FBW (g) & $43.2 \pm 7.5$ & $46.2 \pm 7.2$ & 0.1 \\
\hline
\end{tabular}

Values represent mean $\pm S D$.

$\mathrm{N}=5$ does and 30 fetuses: placebo group; $\mathrm{N}=5$ does and 34 fetuses sildenafil group. FBW, fetal body weight; HRCP, heart rate change percentage; WCP, weight change percentage. 
Table 2 Number of fetuses and pregnancy loss rate in the different study groups

\begin{tabular}{|c|c|c|c|c|c|}
\hline \multirow[b]{2}{*}{ Medication } & \multirow[b]{2}{*}{ Induction of DH } & \multirow[b]{2}{*}{ Survival at CS } & \multicolumn{3}{|l|}{ Read outs } \\
\hline & & & Histology & $\mu \mathrm{CT}$-arteriograms & Functional measures \\
\hline \multicolumn{6}{|l|}{ Placebo } \\
\hline Does & 9 & 9 & & & \\
\hline DH fetuses & 22 & $19 / 22$ & 6 & 6 & 7 \\
\hline CTR fetuses & 57 & $52 / 57$ & 6 & 6 & $7-5^{*}$ \\
\hline \multicolumn{6}{|l|}{ Sildenafil } \\
\hline Does & 9 & 9 & & & \\
\hline DH fetuses & 23 & $17 / 23$ & 6 & $5 \dagger$ & 5 \\
\hline CTR fetuses & 60 & $54 / 60$ & 6 & 6 & 6 \\
\hline
\end{tabular}

similar in the maternal and fetal circulation, with a peak at $30 \mathrm{~min}$ and concentrations above the maximal target for the first hour. Of note, plasmatic sildenafil concentrations in fetal and maternal plasma $22 \mathrm{~h}$ after the third day of therapy did not differ from those $22 \mathrm{~h}$ after the first injection, indicative that the drug is not accumulating.

Tolerance indices were compared in animals treated with sildenafil or placebo for 7 days. There were no significant differences in WCP, HRCP, fetal loss rate and FBW (table 1). Similarly, the course of the behavioural score was comparable between the sildenafil and placebo-treated does (figure 1C).

Subsequently, 18 does underwent laparotomy on GD23 with surgical creation of $\mathrm{DH}$ in 45 fetuses. Of these, 36 fetuses
(80\%, 19/22 placebo-exposed and 17/23 sildenafil-exposed, $\mathrm{p}=0.4)$ survived until harvesting. In all pups, the presence of a diaphragmatic defect and liver herniation was confirmed at obduction. Out of a total of 106 unoperated littermates alive at harvesting, 37 (19 placebo exposed and 18 sildenafil exposed) were used as CTR for further histological and functional analyses (table 2). FBW did not differ between the four study groups, nor the other fetal tolerance indices (see online supplementary figure S1A). DH fetuses had a significantly lower LBWR compared with CTR $(12.4 \pm 0.5 \%$ vs $25.2 \pm 4.9 \%$, $\mathrm{p}<0.001)$. Sildenafil-exposed DH fetuses had a LBWR within the range of $\mathrm{DH}$ fetuses exposed to placebo (see online supplementary figure S1B).
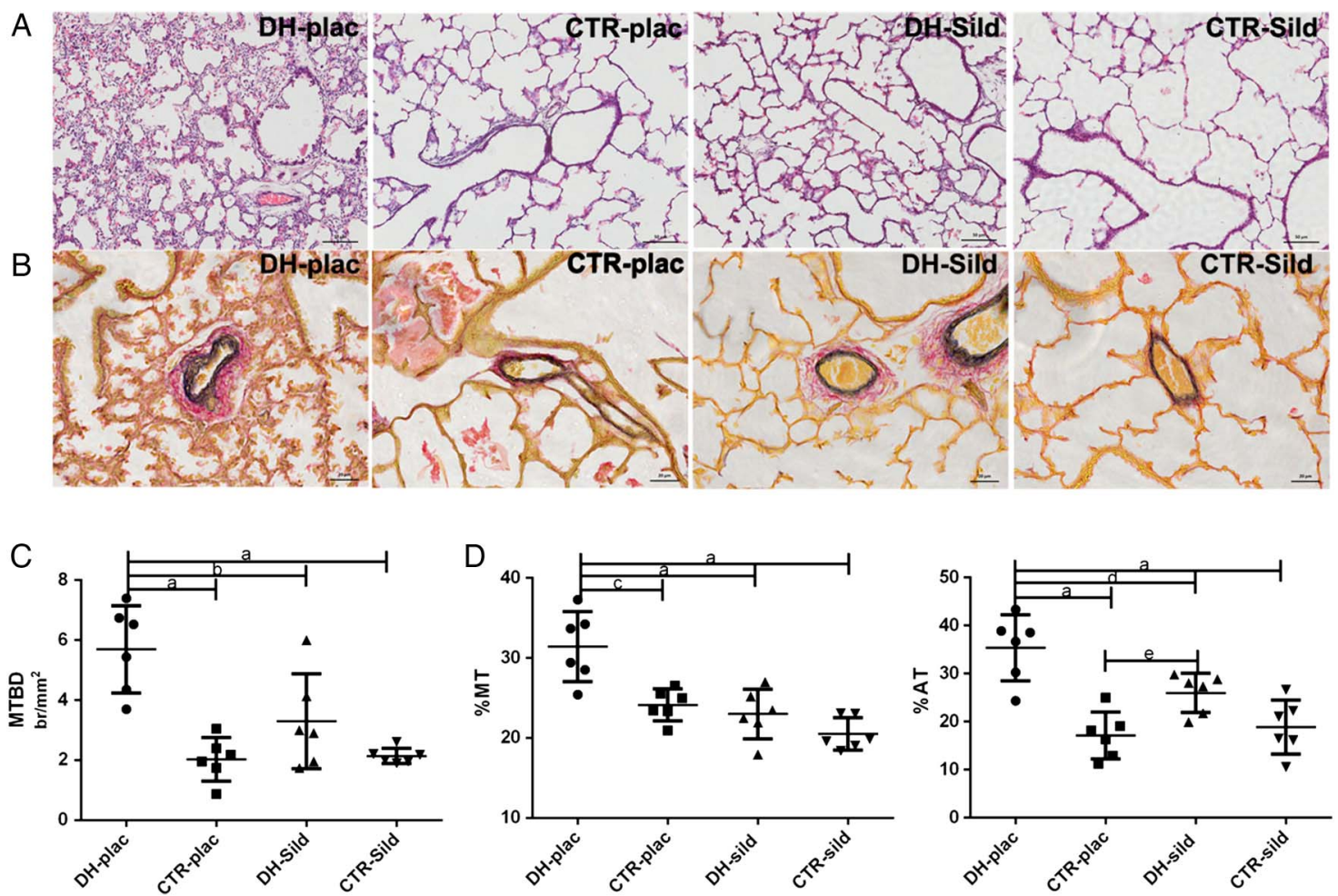

Figure 2 Airway and vascular morphometry. (A and B) Representative H\&E (A) and Miller's (B) stained sections showing differences in lung parenchyma and in medial and adventitial thickness in the four study groups. (C) Mean terminal bronchiolar density (MTBD). (D) Proportionate medial thickness (\%MT) and proportionate adventitial thickness (\%AT). Scale bar: $20 \mu \mathrm{m}$. Large horizontal bars in the middle indicate mean, error bars indicate SD. Significant post hoc corrected $p$ values are reported above comparisons: $a: p<0.001, b: p=0.008, c: p=0.002, d: p=0.034$, e: $p=0.049$. CTR, control group; $D H$, diaphragmatic hernia; plac, placebo; sild, sildenafil. 
Sildenafil reverses the parenchymal and vascular changes of DH

Airway morphometric measures were comparable in $\mathrm{DH}$ and CTR fetuses with the exception of MTBD, a measure of distal airway complexity, which was increased in DH fetuses treated with placebo. Sildenafil-exposed DH fetuses had an MTBD within the range of CTR fetuses (figure 2A, C). Vascular morphometric indices are displayed in figure $2 \mathrm{~B}$, D. The \%MT and proportionate adventitial thickness (\%AT) were significantly higher in DH pups compared with CTR, yet fell in the normal range when DH fetuses were exposed to sildenafil. The differences were more pronounced in vessels with an external diameter (ED) $\leq 60 \mu \mathrm{m}$, which correspond to the intra-acinar or resistance vessels ${ }^{30}$ (see online supplementary figures S2A and S2B). The drug had no effect on vascular indices in CTR pups.

Sildenafil reduces peripheral vascular muscularisation and increases parenchymal Vascular Endothelial Growth Factor (VEGF) expression

DH fetuses had proportionally more muscularised peripheral vessels than CTR (mean \pm SD: $36 \pm 7 \%$ vs $13 \pm 4 \%, \mathrm{p}<0.001$ ) (figure $3 \mathrm{~A}, \mathrm{D}$ ), whereas sildenafil treatment was associated with a degree of peripheral muscularisation in the normal range $(20$ $\pm 2 \%)$. There was no measurable effect of sildenafil on CTR fetuses. Again, these differences were more evident in vessels with an $\mathrm{ED} \leq 60 \mu \mathrm{m}$ (see online supplementary figure $2 \mathrm{C}$ ). Sildenafil was also associated with an increase in the immunoreactivity for VEGF and its receptor Flk-1 in the lung parenchyma, both in DH fetuses and in CTR (figure 3B, C, E, F). The expression of VEGF and Flk-1 in vascular wall cells, instead, did not differ between the four study groups (see online supplementary figure S3).

\section{Sildenafil does not affect vessel volume but restore vessel branching in DH lungs}

Quantification of vessel volume using micro-CT $(\mu \mathrm{CT})$ showed a high variability in the vessel volume/total lung volume ratio, with no significant differences within the four groups (figure 4A). The analysis of vessel branching on reconstructed 3D images demonstrated that the placebo-exposed $\mathrm{DH}$ fetuses had significantly fewer vessels of the fifth order or higher (further called 'peripheral') compared with CTR (mean \pm SD: 18 $\pm 10 \%$ vs $49 \pm 10 \%, \mathrm{p}<0.01)$. The number of peripheral vessels of sildenafil-exposed DH fetuses was in the normal range (43 $\pm 7 \%$ ). In CTR fetuses exposed to sildenafil, instead, the
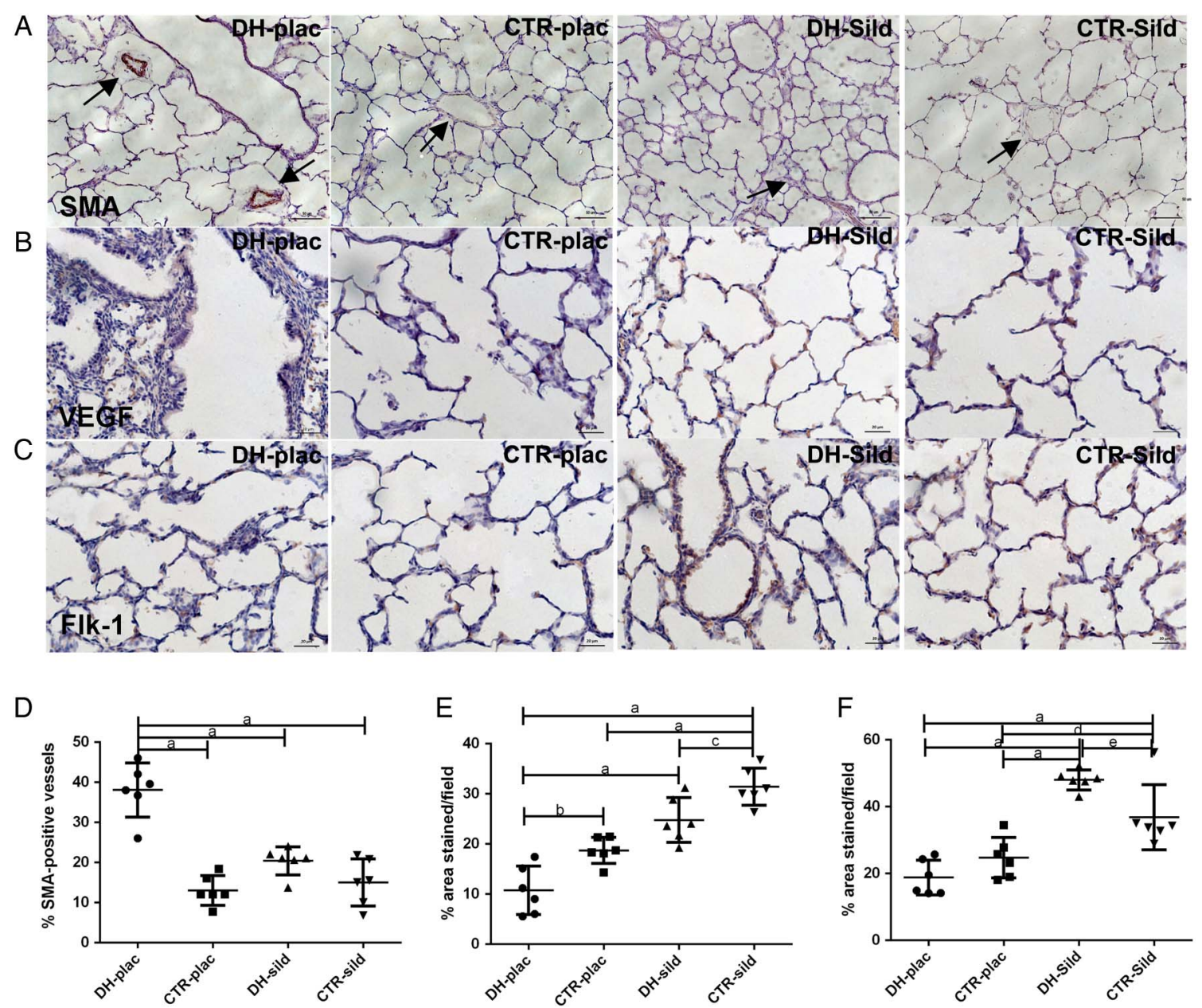

Figure 3 Effect of sildenafil on muscularisation of peripheral vessels and on the VEGF pathway. (A) Representative sections stained for smooth muscle actin (SMA) showing muscularised or partially/non-muscularised vessels. ( $B$ and C) Representative immunohistochemical staining for VEGF (B) and Flk-1 (C) in lung parenchyma. (D) Percentage of SMA-positive vessels in all vessels with external diameter $<100 \mu \mathrm{m}$. (E and F) Quantification of the immunoreactivity for VEGF (E) and Flk-1 (F) in lung parenchyma. Scale bar: $20 \mu \mathrm{m}$. Large horizontal bars in the middle indicate mean, error bars indicate SD. Significant post hoc corrected $p$ values are reported above comparisons: $a: p<0.001, b: p=0.012, c: p<0.042, d: p=0.02, e: p<0.035$. CTR, control group; DH, diaphragmatic hernia; plac, placebo; sild, sildenafil. 

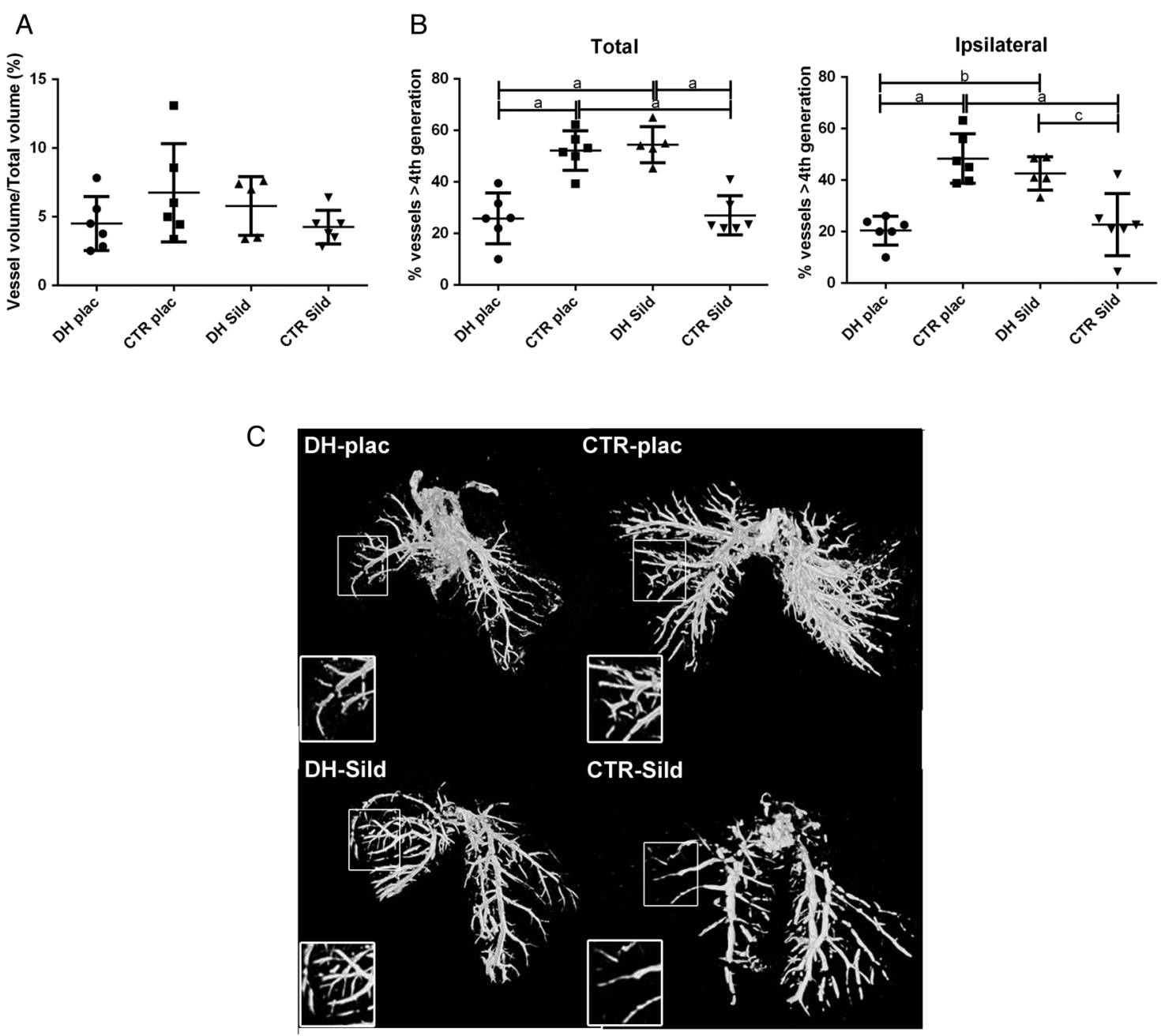

Figure 4 Lung vascular architecture. (A) Vessel volume/total lung volume ratio. (B) Proportion of vessels of fifth order or higher in both lungs and in the left lung. (C) Representative three-dimensional surface rendered images showing impaired vascular branching in DH fetuses, which is restored by exposure to sildenafil. Inserts depict magnified middle lobe of the ipsilateral lung. Large horizontal bars in the middle indicate mean, error bars indicate SD. Significant post hoc corrected $p$ values are reported above comparisons: $a: p<0.001, b: p=0.003, c: p=0.008$. CTR, control group; $\mathrm{DH}$, diaphragmatic hernia; plac, placebo; sild, sildenafil.

percentage of peripheral vessels was not different from placebo-exposed DH fetuses $(23 \pm 11 \%)$. These differences were present in both lungs as well as in the ipsilateral (most hypoplastic) lung (figure $4 \mathrm{~B}, \mathrm{C}$ ).

\section{Sildenafil has a measurable effect on non-invasive pulmonary vascular flow measurement}

None of the measured ECG morphological and Doppler parameters differed between DH and CTR fetuses (data not shown). However, both DH and CTR fetuses exposed to sildenafil had an increased acceleration time/ejection time (AT/ET) ratio in the main pulmonary artery Doppler compared with placebo-exposed DH and CTR fetuses, indicating a vasodilatory effect of the drug on the fetal pulmonary circulation in exposed fetuses (figure $5 \mathrm{~A}, \mathrm{~B}$ ).

\section{Sildenafil improves lung mechanics in DH fetuses}

In placebo-exposed DH fetuses, static compliance was significantly reduced and static elastance significantly increased compared with CTR. Also, DH fetuses had a significantly decreased total lung capacity. In $\mathrm{DH}$ fetuses exposed to sildenafil, these parameters were within the normal range. Of note, this study group presented a higher variability compared with the others in all measured parameters (figure 5C).

\section{DISCUSSION}

In the present study, we demonstrate that, in the rabbit model for $\mathrm{DH}$, maternal sildenafil administration rescues parenchymal and vascular lung abnormalities induced by $\mathrm{DH}$ without any obvious side effect, except for fetuses with normally developed lungs in whom it seems to decrease vascular branching.

We chose the rabbit because it has a lung development similar to man. Unlike rats, humans and rabbits alveolize in utero. ${ }^{29}$ When in rabbits $\mathrm{DH}$ is induced in the pseudoglandular phase, it reproduces the clinical phenotype in various aspects. ${ }^{22} 31$ The rabbit model also allows more detailed assessment of fetomaternal safety, because placental transfer in the latter half of pregnancy mirrors that in humans. ${ }^{32}$ The rabbit mid-gestational and end-gestational placenta is functionally positioned between rodents (hemotrichorial) and man (hemomonochorial). ${ }^{32}$ Also, pregnancy-induced haemodynamic changes are comparable with those present in women, that is, with a steady increase in maternal blood pressure in the latter half of gestation. ${ }^{33}$

In the first part of our study, we assessed the pharmacokinetics of sildenafil in normal pregnant rabbits. We used a 

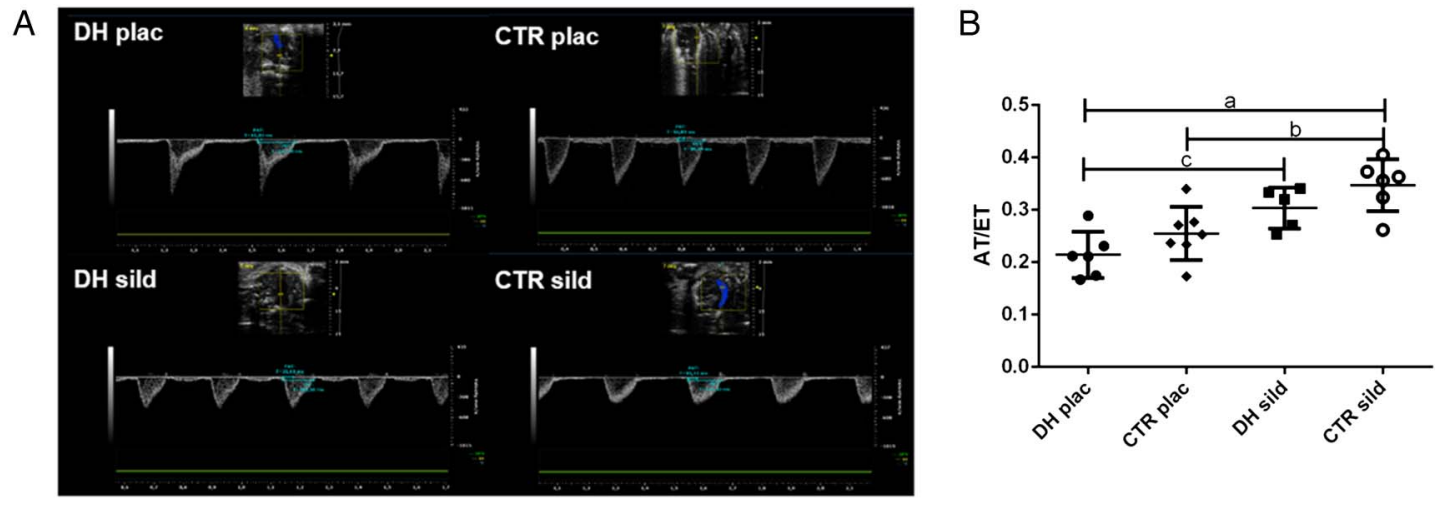

Figure 5 Functional measurements. (A) Representative images of the Doppler waveforms of the main pulmonary artery. (B) Acceleration time/ ejection time (AT/ET) ratio; $\mathrm{N}=5$ per DH-sildenafil, 6 per CTR sildenafil, 7 per the placebo-exposed DH fetuses and placebo-exposed CTR groups. (C) Measures of lung mechanics: total lung capacity, static compliance and static elastance. Large horizontal bars in the middle indicate mean, error bars indicate SD. Significant post hoc corrected $p$ values are reported above comparisons: $a: p<0.001, b: p=0.01, c: p=0.023, d: p=0.002$, e: $p=0.004$, $f: p=0.003$. CTR, control group; $D H$, diaphragmatic hernia; plac, placebo; sild, sildenafil.

comprehensive number of maternal as well as fetal safety measures when testing dosages and administration regimens. We did not observe obvious clinical side effects with $10 \mathrm{mg} / \mathrm{kg} /$ day even though this dose was associated with a short period of abovetarget sildenafil levels. We also excluded drug accumulation after serial administration, a process that otherwise with chronic use could result in toxicity. Thus, we corroborated the aforementioned data from the rodent model, that is, that sildenafil is well tolerated by fetuses in the later stages of gestation. ${ }^{14}$

In addition to this, we investigated the effects of sildenafil treatment on the developing lung. The drug had an effect on parenchymal development with significant improvement in MTBD. The interactions between the airways and blood vessels are critical during lung development. ${ }^{34}$ Therefore, it is postulated that antenatal interventions designed to prevent vascular remodelling may rescue vascular and airway changes. ${ }^{14} 35$ The functional analysis of the lungs with forced oscillation technique parallels the histological findings. As previously experimentally and clinically demonstrated, lung mechanics are significantly compromised in $\mathrm{DH}^{31}$ We showed that such changes are reverted or at least attenuated by sildenafil, though with a high variability among fetuses. Nevertheless, other relevant parameter of lung function (like tissue damping or hysteresivity) could not be extensively investigated due to technical difficulties related to DH lung fragility. Thus, it is possible that parenchymal lung growth is still not ideal as there was no major concomitant increase in LBWR.

The vascular changes associated with $\mathrm{DH}$ are known to induce PPHT. Although PPHT is best known as a postnatal event in $\mathrm{DH}$, the structural changes are already present in utero. $^{22} 36$ The potential benefit of sildenafil on the pathophysiology of PPHT has already been demonstrated in the nitrofen rat model. ${ }^{14}$ In the rabbit, sildenafil reversed the pathological changes in peripheral vessels and improved vascular branching in DH. At the structural level, sildenafil restored vascular wall thickness and muscularisation of peripheral arteries to normal levels. We also performed immunohistochemical analysis for VEGF and for its receptor Flk-1, but in the vasculature we did not detect a difference between the four cohorts. Yet in the alveolar parenchyma, sildenafil increased VEGF-positive and Flk-1-positive cell density both in DH and CTR lungs. VEGF has been shown to interact with the nitric oxide (NO) pathway, by inducing release of $\mathrm{NO}$ from vascular endothelial cells and regulating endothelial NO synthase (eNOS) expression. ${ }^{37}$ The activation of eNOS mediated by sildenafil could, in turn, upregulate VEGF expression. $^{38}$

To our knowledge, this is the first study in animal models for $\mathrm{DH}$, where specific methods for quantitative assessment of vessel density and branching have been used. We did so using barium-gelatin angiograms and $3 \mathrm{D}$ reconstruction on $\mu \mathrm{CT}$ images. We observed decreased peripheral vascular branching in DH fetuses, which was reversed by sildenafil. The latter had also a measurable effect on CTR fetuses, where it reduced vascular branching. This is in line with what has been demonstrated in the rat model. ${ }^{14}$ The mechanism behind this finding remains unclear, but may be related to a reduction in pulmonary blood pressure. ${ }^{39}$ This could be beneficial in lungs with increased vascular resistances, but on the other hand it cannot be excluded that it has adverse effect in more normal lungs, such as hypoperfusion and impaired vessel growth in normal lungs. This finding should prompt cautious use of sildenafil treatment in $\mathrm{DH}$ cases with milder forms of lung hypoplasia. 
Finally, we demonstrated that sildenafil causes a measurable vasodilation of the pulmonary vasculature, resulting in an increased AT/ET ratio of the pulmonary artery Doppler waveform. However, we did not observe any difference between DH fetuses and CTR either in this Doppler parameter or in the cardiac structural dimensions. This is in contrast to what was observed in the nitrofen model. ${ }^{36} \mathrm{DH}$ rats had lower pulmonary artery Doppler ET/ET ratios and PI compared with CTR. In that study, Doppler measurements were performed on the contralateral first branch of the pulmonary artery branch as opposed to the main pulmonary artery in our study. This difference may be caused by the measurable impact of shunting via the ductus arteriosus in our measurement.

Our study design, as well as the animal model, has some limitations. These include the lack of vascular assessment of extrapulmonary vessels, in particular in the brain and liver. We neither did perform quantitative PCR analysis of relevant genes involved in the pathway. PCR, however, only allows a quantification of expression levels in the lung as a whole and not in specific structures such as conductive airways, parenchyma or vasculature, unless structures have been previously discriminated by laser-capture microdissection. Nor do we have postnatal outcomes with regard to long-term toxicity of the drug. Also, our assessment methods of pulmonary flow and resistance or structural cardiac impact failed to demonstrate a difference between treatment groups. This is not different from clinical reality, as in humans there is no robustly validated prenatal ultrasound predictor for PPHT. It would also be interesting to evaluate more selective PDE5 inhibitors, like tadalafil. ${ }^{40}$ However, use of tadalafil in the neonatal population is contraindicated due to lack of maturation of the glucuronidation pathway vital for drug metabolism. ${ }^{41}$ Finally, additional studies may be justified to further investigate the relevant pathways involved in the therapeutic effect of the drug, measurement of PPHT (either invasively or non-invasively) and extensive performance of lung function testing. This may be done both in the rabbit model as well as sheep.

In conclusion, we proved that sildenafil is effective in reversing many of the parenchymal and vascular effects of $\mathrm{DH}$ in the fetal rabbit. Our results corroborate the clinical safety of this drug during pregnancy and might pave the way for clinical application of a novel antenatal medical strategy for $\mathrm{DH}$.

Acknowledgements We thank Rieta Van Bree, Catherina Luyten and Lieve Verbist for their technical help in the histological studies.

Contributors FMR designed the study, conducted the experiments, analysed the data and wrote the manuscript. MPE conducted the experiments and analysed the data. AHM, JJ and PDK conducted the experiments. GVV, UH and PV participated in the study design. JT and KA participated in the study design and revised the manuscript. JD designed the study, revised the manuscript and organised the study as overall supervisor.

Funding Our translational research programme is being funded by the Fonds voor Wetenschappelijk Onderzoek Vlaanderen (FWO; JD as clinical researcher; 1.801207; KA 1.800214; GVV as postdoctoral fellow; 12N7615N), the European Commission via its Erasmus Joint Doctoral programme (MPE and JJ; 2013-0040). JT is beneficiary of a clinical research grant from the 'Klinische Opleidings-en Onderzoeks-Raad' of the University Hospitals Leuven.

Competing interests None declared.

Provenance and peer review Not commissioned; externally peer reviewed.

\section{REFERENCES}

1 Kotecha S, Barbato A, Bush A, et al. Congenital diaphragmatic hernia. Eur Respir J 2012;39:820-9.

2 Rottier R, Tibboel D. Fetal lung and diaphragm development in congenital diaphragmatic hernia. Semin Perinatol 2005;29:86-93.
3 Neonatal Inhaled Nitric Oxide Study Group. Inhaled nitric oxide in full-term and nearly full-term infants with hypoxic respiratory failure. $N$ Engl J Med 1997;336:597-604.

4 Hayakawa $M$, Ito $M$, Hattori $T$, et al. Effect of hospital volume on the mortality of congenital diaphragmatic hernia in Japan. Pediatr Int 2013;55:190-6.

5 Reiss I, Schaible T, van den Hout L, et al. Standardized postnatal management of infants with congenital diaphragmatic hernia in Europe: the CDH EURO Consortium consensus. Neonatology 2010;98:354-64.

6 Tovar JA. Congenital diaphragmatic hernia. Orphanet J Rare Dis 2012;7:1.

7 Claus F, Sandaite I, DeKoninck P, et al. Prenatal anatomical imaging in fetuses with congenital diaphragmatic hernia. Fetal Diagn Ther 2011;29:88-100.

8 DiFiore JW, Fauza DO, Slavin R, et al. Experimental fetal tracheal ligation reverses the structural and physiological effects of pulmonary hypoplasia in congenital diaphragmatic hernia. J Pediatr Surg 1994;29:248-56; discussion 56-7.

9 Done' $E$, Lewi $P$, Rayyan $M$, et al. 56: Neonatal morbidity in fetuses with severe isolated congenital diaphragmatic hernia $(\mathrm{CDH})$ in the FETO era. Am J Obstet Gynecol 2011;201:S33.

10 Schermuly RT, Kreisselmeier KP, Ghofrani HA, et al. Chronic sildenafil treatment inhibits monocrotaline-induced pulmonary hypertension in rats. Am J Respir Crit Care Med 2004;169:39-45.

11 Wharton J, Strange JW, Møller GM, et al. Antiproliferative effects of phosphodiesterase type 5 inhibition in human pulmonary artery cells. Am J Respir Crit Care Med 2005;172:105-13.

12 Baquero $H$, Soliz A, Neira $F$, et al. Oral sildenafil in infants with persistent pulmonary hypertension of the newborn: a pilot randomized blinded study. Pediatrics 2006;117:1077-83.

13 Ganzevoort W, Alfirevic Z, von Dadelszen P, et al. STRIDER: Sildenafil Therapy In Dismal prognosis Early-onset intrauterine growth Restriction-a protocol for a systematic review with individual participant data and aggregate data meta-analysis and trial sequential analysis. Syst Rev 2014;3:23.

14 Luong C, Rey-Perra J, Vadivel A, et al. Antenatal sildenafil treatment attenuates pulmonary hypertension in experimental congenital diaphragmatic hernia. Circulation 2011;123:2120-31.

15 Cheitlin MD, Hutter AM Jr, Brindis RG, et al. ACC/AHA expert consensus document Use of sildenafil (Viagra) in patients with cardiovascular disease. American College of Cardiology/American Heart Association. J Am Coll Cardiol 1999;33:273-82.

16 Goldenberg MM. Safety and efficacy of sildenafil citrate in the treatment of male erectile dysfunction. Clin Ther 1998;20:1033-48.

17 Ballard SA, Gingell CJ, Tang K, et al. Effects of sildenafil on the relaxation of human corpus cavernosum tissue in vitro and on the activities of cyclic nucleotide phosphodiesterase isozymes. J Urol 1998;159:2164-71.

18 Barst RJ, Ivy DD, Gaitan G, et al. A randomized, double-blind, placebo-controlled, dose-ranging study of oral sildenafil citrate in treatment-naive children with pulmonary arterial hypertension. Circulation 2012;125:324-34.

19 Vos RM, Chahbouni A, Sinjewel A, et al. Quantitative analysis of sildenafil and desmethylsildenafil in human serum by liquid chromatography-mass spectrometry with minimal sample pretreatment. J Chromatogr B Analyt Technol Biomed Life Sci 2008:876:283-7.

20 Leach MC, Allweiler S, Richardson C, et al. Behavioural effects of ovariohysterectomy and oral administration of meloxicam in laboratory housed rabbits. Res Vet Sci 2009;87:336-47.

21 Keating SC, Thomas AA, Flecknell PA, et al. Evaluation of EMLA cream for preventing pain during tattooing of rabbits: changes in physiological, behavioural and facial expression responses. PLOS ONE 2012;7:e44437.

22 Wu J, Yamamoto $H$, Gratacos $E$, et al. Lung development following diaphragmatic hernia in the fetal rabbit. Hum Reprod 2000;15:2483-8.

23 Roubliova XI, Lewi PJ, Vaast P, et al. Effects of betamethasone on peripheral arterial development in term fetal rabbit. Pediatr Pulmonol 2008;43:795-805.

24 De Langhe E, Vande Velde G, Hostens J, et al. Quantification of lung fibrosis and emphysema in mice using automated micro-computed tomography. PLOS ONE 2012;7:e43123.

25 Counter WB, Wang IQ, Farncombe TH, et al. Airway and pulmonary vascular measurements using contrast-enhanced micro-CT in rodents. Am J Physiol Lung Cell Mol Physiol 2013;304:L831-43.

26 Rychik J, Ayres N, Cuneo B, et al. American Society of Echocardiography guidelines and standards for performance of the fetal echocardiogram. J Am Soc Echocardiogr 2004; 17:803-10.

27 Hodges R, Endo M, La Gerche A, et al. Fetal echocardiography and pulsed-wave Doppler ultrasound in a rabbit model of intrauterine growth restriction. J Vis Exp 2013;(76):50392.

28 Jani JC, Flemmer AW, Bergmann F, et al. The effect of fetal tracheal occlusion on lung tissue mechanics and tissue composition. Pediatr Pulmonol 2009;44:112-21.

29 Roubliova XI, Deprest JA, Biard JM, et al. Morphologic changes and methodological issues in the rabbit experimental model for diaphragmatic hernia. Histol Histopathol 2010;25:1105-16.

30 Roubliova XI, Verbeken EK, Wu J, et al. Effect of tracheal occlusion on peripheric pulmonary vessel muscularization in a fetal rabbit model for congenital diaphragmatic hernia. Am J Obstet Gynecol 2004;191:830-6. 
31 Flemmer AW, Jani JC, Bergmann F, et al. Lung tissue mechanics predict lung hypoplasia in a rabbit model for congenital diaphragmatic hernia. Pediatr Pulmonol 2007;42:505-12.

32 Fischer B, Chavatte-Palmer $\mathrm{P}$, Viebahn $\mathrm{C}$, et al. Rabbit as a reproductive model for human health. Reproduction 2012;144:1-10.

33 McArdle AM, Denton KM, Maduwegedera D, et al. Ontogeny of placental structural development and expression of the renin-angiotensin system and 11 beta-HSD2 genes in the rabbit. Placenta 2009:30:590-8.

34 Stenmark KR, Abman SH. Lung vascular development: implications for the pathogenesis of bronchopulmonary dysplasia. Annu Rev Physiol 2005;67: 623-61.

35 Deprest J, Gucciardo L, Eastwood P, et al. Medical and regenerative solutions for congenital diaphragmatic hernia: a perinatal perspective. Eur J Pediatr Surg 2014;24:270-7.
36 Yamamoto Y, Thebaud B, Vadivel A, et al. Doppler parameters of fetal lung hypoplasia and impact of sildenafil. Am J Obstet Gynecol 2014;211:263.e1-8.

37 Frelin C, Ladoux A, D'Angelo G. Vascular endothelial growth factors and angiogenesis. Ann Endocrinol (Paris) 2000;61:70-4.

38 Zhao X, Lu X, Feng Q. Deficiency in endothelial nitric oxide synthase impairs myocardial angiogenesis. Am J Physiol Heart Circ Physiol 2002;283:H2371-8.

39 Reffelmann T, Kloner RA. Therapeutic potential of phosphodiesterase 5 inhibition for cardiovascular disease. Circulation 2003;108:239-44.

40 Shue EH, Schecter SC, Gong W, et al. Antenatal maternally-administered phosphodiesterase type 5 inhibitors normalize eNOS expression in the fetal lamb model of congenital diaphragmatic hernia. J Pediatr Surg 2014;49:39-45; discussion 45.

41 Vorhies EE, Ivy DD. Drug treatment of pulmonary hypertension in children. Paediatr Drugs 2014;16:43-65. 